\title{
Hypergraph-Based Model for Coexistence Management of Heterogeneous Wireless Networks
}

\author{
$1^{\text {st }}$ Tawachi Nyasulu \\ Department of Electronic and Electrical Engineering \\ University of Strathclyde \\ Glasgow, United Kingdom \\ Email: tawachi.nyasulu@strath.ac.uk
}

\author{
$2^{\text {nd }}$ David H. Crawford \\ Department of Electronic and Electrical Engineering \\ University of Strathclyde \\ Glasgow, United Kingdom \\ Email: david.crawford@strath.ac.uk
}

\begin{abstract}
Traditional graph theory is typically used to model interference relations among networks to realise channel assignment that enables improvement in spectrum utilisation through spatial re-use of the channels. Studies have shown that spectrum utilisation could be further improved through co-sharing among networks that are capable of spectral coexistence as long as the channel load is not excessive. The co-sharing networks use their inherent media access control (MAC) techniques to coordinate access to a shared channel. However, the concept of an edge in a traditional graph, which is a two-element subset, is not sufficient to model subsets of potential co-sharing networks because such subsets may have cardinality of greater than 2 . Instead, this paper proposes use of hypergraph theory to model the co-sharing strategy in an environment that comprises heterogeneous radio systems. The model could be applied in centralised coexistence management frameworks such as IEEE 802.19.1-based systems. Results demonstrate that spectrum sharing using the hypergraph model achieves higher average spectrum utilisation by up to $17.5 \%$ when there are 3 available channels, and requires up to 7 fewer channels to achieve, on average, $100 \%$ operational networks than spatial re-use alone.
\end{abstract}

Index Terms-coexistence management, spectral coexistence, radio resource allocation, hypergraph theory, IEEE 802.19.1, television white space

\section{INTRODUCTION}

The electromagnetic spectrum, which is a limited resource, is one of the major limitations on the growth of wireless communication networks. Therefore, policies and techniques for efficient management of spectrum are important. Dynamic Spectrum Access (DSA) is regarded as a promising solution for efficient spectrum management. DSA policy allows secondary users to access the spectrum of licensed (primary) users either on licence-exemption or on temporary exclusive licence bases, when the spectrum is not being used by the primary users.

The US, the UK and a few other countries have approved DSA policy in the television (TV) band and the vacant spectrum in this band is known as Television White Space (TVWS). Whereas primary users, that is TV stations and wireless microphones, are guaranteed protection from interference, secondary users are not protected. Licence-exempt spectrum performance degradation as a result of interference among the secondary user networks when the amount of available channels is not enough to allow exclusive channel operation for each network. The coexistence challenge among secondary user networks is exacerbated by the presence of heterogeneous radio access technologies (RATs) and lack of coordinated radio resource allocation among the secondary users themselves. The gains in spectrum utilisation as a result of DSA policy can potentially be diminished by the impact of interference.

Spectrum utilisation can be improved by spatial reuse of channels. However, licence-exempt spectrum is likely not to be enough for exclusive channel operation, especially in urban areas. Hence, spectrum utilisation may be further improved through co-sharing among neighbour networks that can effectively coordinate access to a shared channel, as long as the total channel occupancy rate (COR) is less than unity to prevent spectrum starvation [1]. This paper therefore focuses on the design of a radio resource allocation framework for maximising spectrum utilisation through spectrum sharing, while ensuring coexistence among heterogeneous secondary user networks.

In this paper, heterogeneous networks refers to networks that use different RATs. Such networks may not effectively coordinate access to a shared spectrum due to incompatible wireless protocols. The IEEE 802.19.1 standard specifies high level, network-based coexistence methods for geo-locationcapable devices operating under general authorization access (GAA) or licence-exemption. However, actual implementation of modelling techniques and radio resource allocation algorithms of the coexistence system is left to the industry [2].

A hypergraph is a generalization of an undirected graph in which a hyperedge is a subset of vertices of arbitrary cardinality [3]. A radio frequency (RF) environment can be represented by a hypergraph, whereby radio devices or networks are represented by vertices and properties of different subsets are represented by hyperedges. Use of hypergraphs for modelling of cumulative interference and network dependency properties in radio resource allocation schemes has been proposed in literature [4]-[7].

This paper proposes application of hypergraph theory in modelling of spectral coexistence capabilities of radios in a heterogeneous RF environment in order to implement a co- 
sharing strategy. The paper further proposes use of hyperedge contraction to decompose a hypergraph, whereby the vertices of a hyperedge are replaced by one vertex without losing information about other relationships, in order to make the graph amenable to traditional graph algorithms.

Application of the model is demonstrated for implementation of coordinated channel allocation and coexistence management for IEEE 802.19.1-based centralised coexistence management system in TVWS networks. Performance of the proposed radio resource allocation model is simulated to study the extent to which the spectrum sharing algorithm improves spectrum utilisation beyond spatial re-use. Implementation of the model for other DSA systems, such $3.5 \mathrm{GHz}$ Citizen Broadband Radio Service (CBRS) will be explored in future work.

This paper is organised as follows. Section II is a brief review of previous work. The system model is described in Section III. A channel assignment algorithm based on spatial re-use using traditional graph theory is described in Section IV. The hypergraph-based method is presented in Section V. Simulation results are provided in Section VI. Finally, conclusions and future work are drawn in Section VII.

\section{RELATED WORK}

Research on radio resource allocation algorithms has focused on two main objectives: to maximise spectrum utilisation and to minimise interference among networks. Traditional graph theory is typically used to represent interference relations among networks. Vertices of the graph represent cells and edges exist between vertices of cells that have potential to interfere when operating on the same channel. Vertex colouring algorithm is then used to solve the channel allocation problem whereby a colour assignment of a vertex represents a channel assignment. To minimise interference, vertices that have an edge between them are assigned different colours.

An implementation of coordinated spectrum allocation and coexistence management for GAA users in CBRS networks was proposed in [8]. Graph theory was used to represent interference relationships and channel assignment in space and frequency. Spectrum re-usability was improved through consideration of aggregated interference and transmit power reduction. In [9], traditional graph was used to model channel assignment in space and frequency domain for networks operating in the $2.4 \mathrm{GHz}$ ISM band. Spectrum utilisation was further improved through transmission power control. An evolutionary algorithm was used to optimise radio resource allocation. Spectrum sharing in the time domain was not considered because time synchronisation among heterogeneous network is a challenge.

Co-channel operation among networks that use Time Division Multiple Access (TDMA)-based MAC mechanisms requires time-synchronised operation among the networks. Synchronisation can happen over wireless connection if the network controllers are within each other's communication range and if they use compatible RATs. Similarly, networks that use contention-based MAC mechanisms, such as Carrier Sense Multiple Access/Collision Avoidance (CSMA/CA), need to be within each other's wireless range to be able to detect each other's transmissions. Thus, these conditions can be evaluated to derive groups of networks that can manage interference among themselves when they share a channel.

In [1], a spectrum sharing approach for TVWS is proposed. Exclusive channel allocation based on spatial coexistence is first applied, and if a base station can not find an unoccupied channel which has not been taken already by its neighbours, it shares a channel with neighbour networks of the same RAT. The limitation of this approach is that only RAT compatibility is used as the criteria for spectral coexistence. It is assumed that master devices of neighbour networks are within communication range to effectively coordinate access to shared channels, or an IP connection is used instead. Furthermore, with this approach, the spectrum sharing options could be limited by the outcome of the initial exclusive channel allocation.

Building on the previous work, this paper proposes an alternative approach, whereby information about groups of coexistent networks is integrated in the model so that channels are assigned to groups rather than to individual networks. Communication range between master devices of neighbour networks is also included in the criteria for coexistence analysis.

The concept of an edge in a traditional graph is not sufficient to model multiple relations. On the other hand, a hypergraph is a generalization of an undirected graph in which a hyperedge is a subset of vertices of arbitrary cardinality rather than twoelement subsets [3]. Hypergraph theory has found application in radio resource allocation schemes for shared spectrum environments whereby a hyperedge is used to model multiple relationships.

Hypergraph-based models have been proposed for implementing interference-aware radio resource allocation that alleviates not only interference between pairs of nodes, but also cumulative interference from multiple nodes, in vehicleto-everything (V2X) and device-to-device (D2D) underlay communications, and in dense femtocell networks [4]-[7]. A node or a network is represented by a vertex. A pair of vertices are connected by an edge if the radios or networks that are represented by the vertex pair have potential to interfere when operating in the same channel. A hyperedge represents a subset of neighbouring nodes or networks whose cumulative interference exceeds a set threshold. In this application, resource allocation problem is solved using a strong hypergraph colouring algorithm to ensure that vertices that are incident to the same edge and/or hyperedge cannot be coloured by the same colour to alleviate cumulative interference.

A hypergraph-based model for spectrum management that ensures coexistence management among heterogeneous wireless by frequency-isolating networks that use incompatible media access technologies is proposed in [10]. A hypergraph model is used to represent the structure of the RF environment such that the hypergraph can be searched for 
specific relations among the radios and conflict subgraphs can be derived, in order to realise spectrum assignment that ensures coexistence of heterogeneous wireless networks. In this model, a vertex represents a radio device, a hyperedge represents radios that belong to the same network and thus require the same channel, and an edge between a pair of vertices represents a transmission link if the vertices belong to the same hyperedge, otherwise it represents an interference relationship. The channel assignment problem is modelled as a mixed-integer program (MIP) that uses the relationships from the hypergraph model to derive constraints and the conflict subgraphs and measurement metrics to optimise the channel assignment.

This paper proposes an alternative approach to implementing a co-sharing resource allocation scheme that is both interference-aware and spectral coexistence-aware. A vertex represents a network cell, an edge between a pair of vertices represents interference relationships and a hyperedge represents groups of coexistent networks, such that the channel allocation algorithm is able to assign channels to groups of coexistent networks instead of individual networks. Implementation of the solution is designed for multi-network and/or multioperator coexistence coordination systems such as for GAA secondary user networks in TVWS and CBRS. Nonetheless, the concept can generally be applied, with slight modification, to other network management and frequency planning problems which are characterised by multiple relationships and which could be easier to solve by characterising and decomposing multiple relationships using hypergraph theory.

\section{System Model}

The test scenario is illustrated in Fig. 1 . There are $N$ wireless networks operating using the following RATs: IEEE 802.22 wireless regional area network (WRAN) and 802.11 television very high throughput (TVHT) wireless local area network (WLAN). Each base station (BS) and access point (AP) radio supports a set of slave devices in the form of customer premise equipment (CPEs) and user devices (UEs), respectively. In a cell, the master device and the slave devices communicate on the same channel and the master device coordinates access to the channel among its slave devices.

The networks may not be able to communicate directly with each other by using their MAC/PHY protocols either because they use incompatible network technologies or they are outside the communication range. The geo-location, service area, receiver sensitivity and RAT of each master device is known. A service area or coverage area of a network is specified by its coverage radius, with reference to the location of the master device. Slave devices are located within the service area.

The framework is based on the TVWS regulatory framework that has been approved by UK's Office of Communication (Ofcom) [11]. The master devices have access to a regulatorapproved geo-location spectrum database (GLSD) via IP backhaul connection. The GLSD provides the list of TV channels that are available at the location of a master device. Thus, primary user protection is not considered in this system design

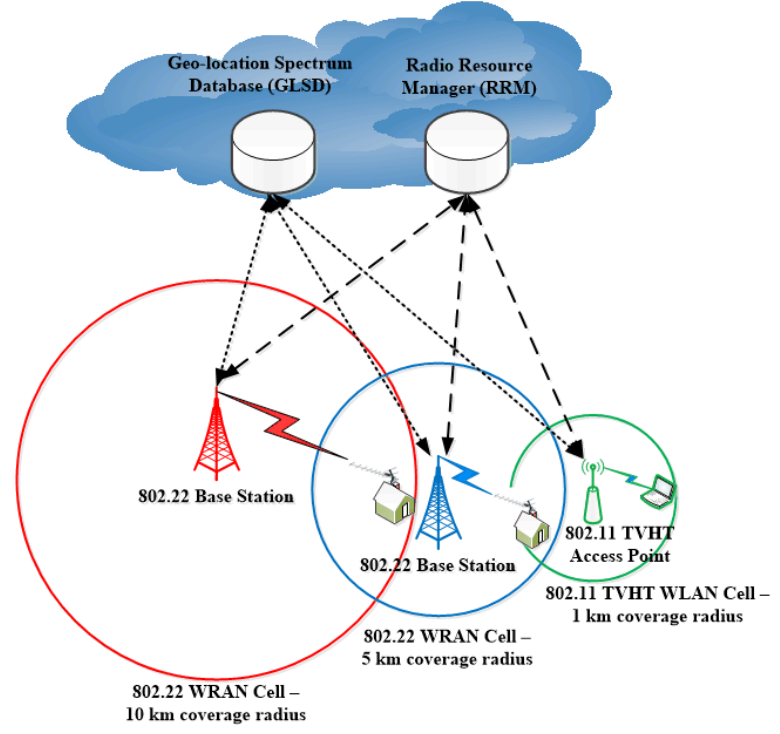

Fig. 1. System model for heterogeneous networks operating in TVWS under the control of a GLSD and an RRM

because primary users are protected by the GLSD. The master device chooses an operating channel from the list and reports its channel usage parameters to the database before it starts operation.

The master device also has access to an IEEE 802.19.1based centralised radio resource manager (RRM), which is not part of Ofcom's TVWS framework, but the service can be provided by a third-party such as a GLSD operator. The master device provides the RRM with its operating channel and the list of available channels. The RRM runs the radio resource allocation algorithm and requests the master device to reconfigure its network accordingly. The assigned frequency and transmission power must comply with the output of the GLSD. The master device periodically contacts the GLSD to check the validity of its operating parameters.

\section{A. Interference Analysis}

It is assumed that all networks have overlap in their operating frequency capabilities and therefore all networks have potential to operate in the same frequency and to interfere. Only co-channel interference is considered. Adjacent Channel Leakage Ratio (ACLR) performance of TVWS radios is regulated in order to prevent interference to licensed primary users. Interference levels are calculated for the worst case scenario when the master device is transmitting at maximum power permissible by regulation and/or attainable by the radio technology standards, and at the longest wavelength for the lowest frequency where TVWS is permitted in the TV band. Transmission power control for optimisation of spectrum reusability, which has been studied extensively in previous work, is out of the scope of this paper.

Only interference caused by the master device is used in determining interference relations among cells because the master device, which usually has an omni-directional antenna 


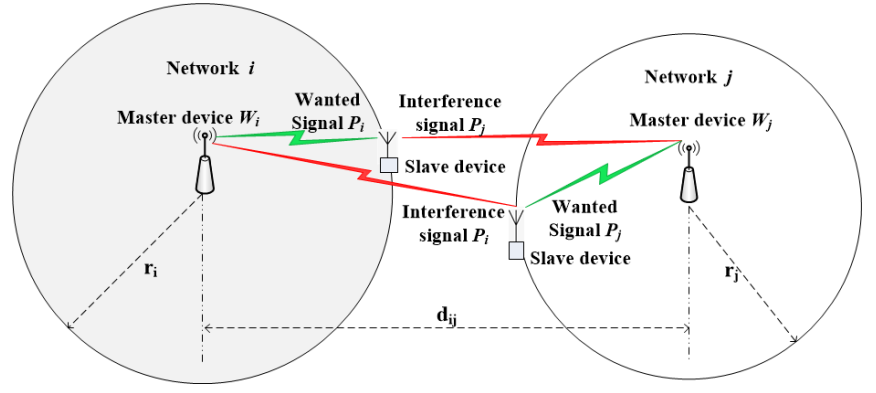

Fig. 2. Interference analysis between a pair of networks with non-overlapping cover areas using the ratio of the wanted signal to the interference signal.

and transmits at a higher power, is more likely to cause or receive interference than slave devices. Even though CPEs use directional antennas, CPEs that are within the line of sight of more than one $\mathrm{BS}$ will cause or receive interference. It is assumed that this scenario will be avoided at the time of CPE installation since the IEEE 802.22.2 Standard for Installation and Deployment of IEEE 802.22 Systems specifies that the CPE antenna should be oriented toward its serving BS and should be further adjusted to minimise the gain in the direction of an interfering BS while keeping the gain toward its serving BS within $2 \mathrm{~dB}$ of its maximum. Nonetheless, such BSs are likely to have overlapping coverage/interference areas and would therefore be evaluated as potential interferers.

Consider two cells, $i$ and $j$, with master devices $W_{i}$ and $W_{j}$, and with coverage radii $r_{i}$ and $r_{j}$. Let $d_{i j}$ be the physical distance between the two master devices that is calculated from their geo-locations using the Haversine Formula. Two conditions are used as criteria for interference analysis. A pair of networks are considered as neighbours if their coverage areas overlap and this condition is evaluated by eq. 1 .

$$
d_{i j}<r_{i}+r_{j}
$$

If their coverage areas do not overlap, as illustrated in Fig. 2 , the pair of networks are neighbours if the value of the signal-to-interference-plus-noise ratio (SINR), at the edge of its coverage area in either direction, is less than a set threshold, $\delta$. This condition is satisfied if eq. 2 or 3 holds true. Since it would be computationally intensive to calculate the SINR for each slave device, it is sufficient for this model to consider SINR of cell-edge slave devices only because these experience lowest SINR due to their distant locations.

$$
\begin{aligned}
& \frac{P_{i}}{P_{j}}<\delta_{i} ; \text { at the edge of network } i \\
& \frac{P_{j}}{P_{i}}<\delta_{j} ; \text { at the edge of network } j
\end{aligned}
$$

\section{B. Coexistence Analysis}

Multiple IEEE WRAN BSs use on-demand frame contention to share a channel in the time domain. In WLANs, UEs use CSMA/CA with Request to Send/Clear To Send (RTS/CTS) to minimise collisions as specified in the IEEE
1900.7 standard for radio interface for white space dynamic spectrum access radio systems. These two radio standards cannot effectively coexist in the same channel because, while the IEEE 802.11 TVHT devices can back-off when an IEEE 802.22 radio is transmitting, the IEEE 802.22 radio would not back off because it is an 'impolite' radio.

The method for evaluation of coexistence is based on the IEEE 802.19.1 mechanisms for "co-sharing via network geometry classification”, and two classes in which synchronisation happens over the wireless connection are considered.

a) The coverage area of a smaller network is completely overlaid by that of a wider network (Class 4): In such cases, the two networks can share a channel if the interference power from the wider network to the smaller network is not at harmful levels. BS interference into neighbouring BSs can be prevented by avoiding line of sight and/or maximising the separation distances between BSs. In this study, this condition is provided for by specifying a minimum separation distance between the master devices.

The absolute minimum separation between antennas should be a horizontal distance of greater than 1/4 of its wavelength, but it should not be located at the exact multiples of its wavelength. The longest wavelength in the TV band is $0.63247 \mathrm{~m}$ for the centre frequency of the lowest channel, $474 \mathrm{MHz}$. In [12], a controlled study was conducted to analyse performance and coexistence among IEEE 802.15.4, 802.11 and 802.22 radio systems operating in TVWS in geographical proximity. The study concluded that generally, all of the systems would be significantly degraded if the interferer is located within $12 \mathrm{~m}$ range. Hence, a separation distance of $15 \mathrm{~m}$ is used in this model. Networks $i$ and $j$ are considered as coexisting networks if (4) is satisfied.

$$
d_{i j}<r_{i} \text { or } d_{i j}<r_{j} \text { and } d_{i j}>15 m
$$

b) The coverage areas of $W_{i}$ and $W_{j}$ overlap and the two master devices are within each other's communication range (Class 1): This class is satisfied if equations (1), and (5) and (6) hold true. The noise floor, $N$, is based on the sensitivity of the receive antenna.

$$
\frac{P_{i}}{N}>\delta_{j} ; \text { at the geo-location of master device } W_{j}
$$

$\frac{P_{j}}{N}>\delta_{i}$; at the geo-location of master device $W_{i}$

\section{Graph-BAsed ExClusive Resource Allocation}

Interference information is represented by an undirected graph $G=(V, E)$ using adjacency lists. Construction of the interference graph is described in Algorithm 1.

The vertex colouring problem seeks to colour the vertices of $V$ using the minimum number of colours such that all neighbour vertices are coloured using different colours, that is: $v_{i}$ and $v_{j}$ have different colours for all $\left(v_{i}, v_{j}\right) \in E$. Thus, a cell is assigned an exclusive channel among its neighbour 


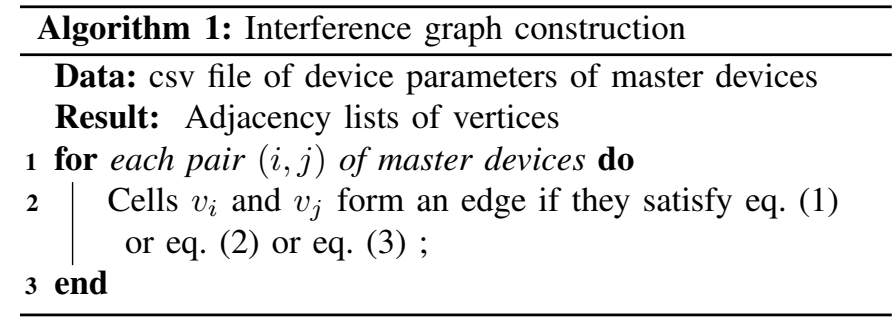

cells. The degree of a vertex is the number of edges that are incident to the vertex. Nodes are coloured in descending order of vertex degree since high degree vertices represent the networks that interfere with more networks and therefore have more colour constraints. Where there is a tie, the vertices are coloured in ascending order of network identity number. The pseudocode is given in Algorithm 2.

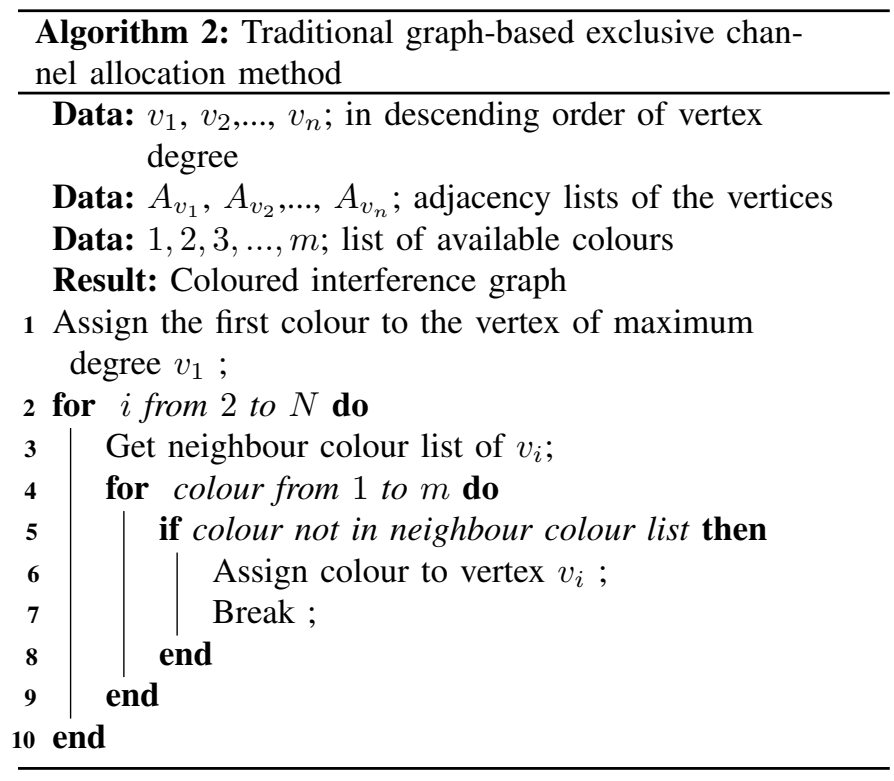

\section{HyPERGRAPH-BASED MODEL}

Since the hyperedges represent subsets of any cardinality, not just 2 as in graphs, hyperedges can be used to represent "arbitrary general statements about arbitrary subsets" [3]. An example of a hypergraph is illustrated in Fig. 3.

Let $H=(V, E)$ be a hypergraph, let $V=\left\{v_{1}, v_{2}, \ldots, v_{n}\right\}$ be a set of vertices and let $E=\left\{e_{1}, e_{2}, \ldots, e_{m}\right\}$ be a set of hyperedges of the hypergraph $H$ such that:

$$
e_{j} \neq \emptyset \text { and } \bigcup_{i=1}^{m} e_{j}=V
$$

Any two hyperedges are said to be adjacent if the intersection of their sets is not an empty set. Contraction of hyperedges is widely used in many algorithms to decompose a hypergraph [3]. Let $e$ be a hyperedge in a hypergraph $H=(V E)$. A contraction of the hyperedge $e$, as illustrated if Fig. 3, involves the following two steps:

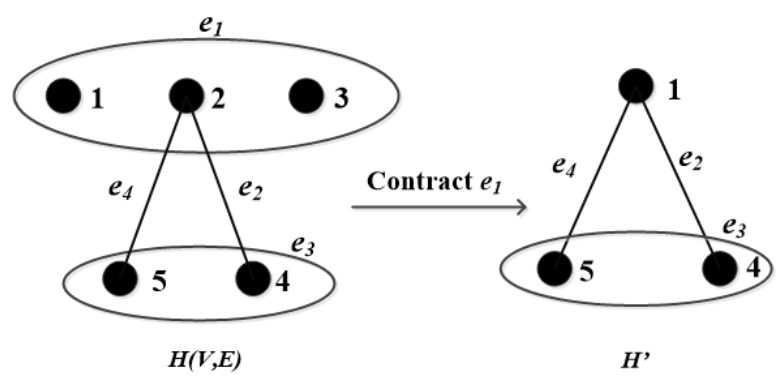

Fig. 3. Illustration of a hypergraph and contraction of hyperedge $e_{1}$.

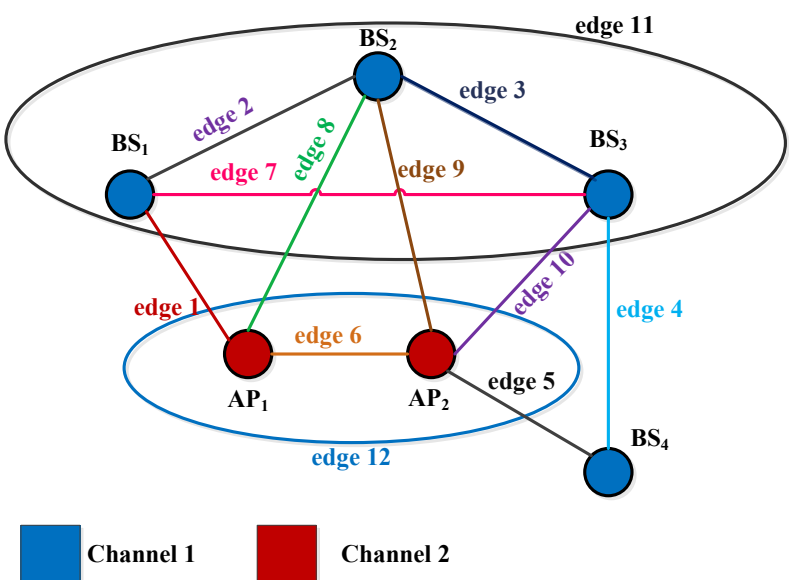

Fig. 4. An example of hypergraph modelling.

- weakly delete $e$ from $H$.

- replace all vertices of $e$ by one vertex belonging to each $e^{\prime}$ such that $e^{\prime} \cap e \neq \emptyset$

\section{A. Hypergraph Construction}

In this model, hypereges are used to represent subsets of vertices that represent cells whose master devices can effectively coexist in the same operating channel using compatible RATs and the total channel load among the cells is less than $100 \%$. An example of this hypergraph modelling is given in Fig. 4. Edges 1 to 10 represent interference relations. When there are only 2 available channels, the exclusive channel allocation algorithm described in Section IV would assign operating channels to only 4 out of the 6 radios. Edges 11 and 12 are hyperedges that represent coexistent subsets. A spectrum sharing algorithm would assign channels 1 and 2 to hypereges 11 and 12 respectively, and $B S_{4}$ would be assigned channel 1 because it could coexist with its neighbour $B S_{3}$. Thus, all 6 radios would be assigned an operating channel.

To construct the hypergraph, the first step is to construct the edges between every pair of the vertices that have potential to interfere with each other if they transmit on the same channel. Algorithm 1 is used. Next, coexistence analysis is performed between neighbour networks that operate using the same RAT. 
The procedure is described in Algorithm 3. The output is an $N \times N$ coexistence matrix $\mathrm{C}$, where an entry of 1 for $C_{i j}$ signifies that networks $i$ and $j$ can coexist.

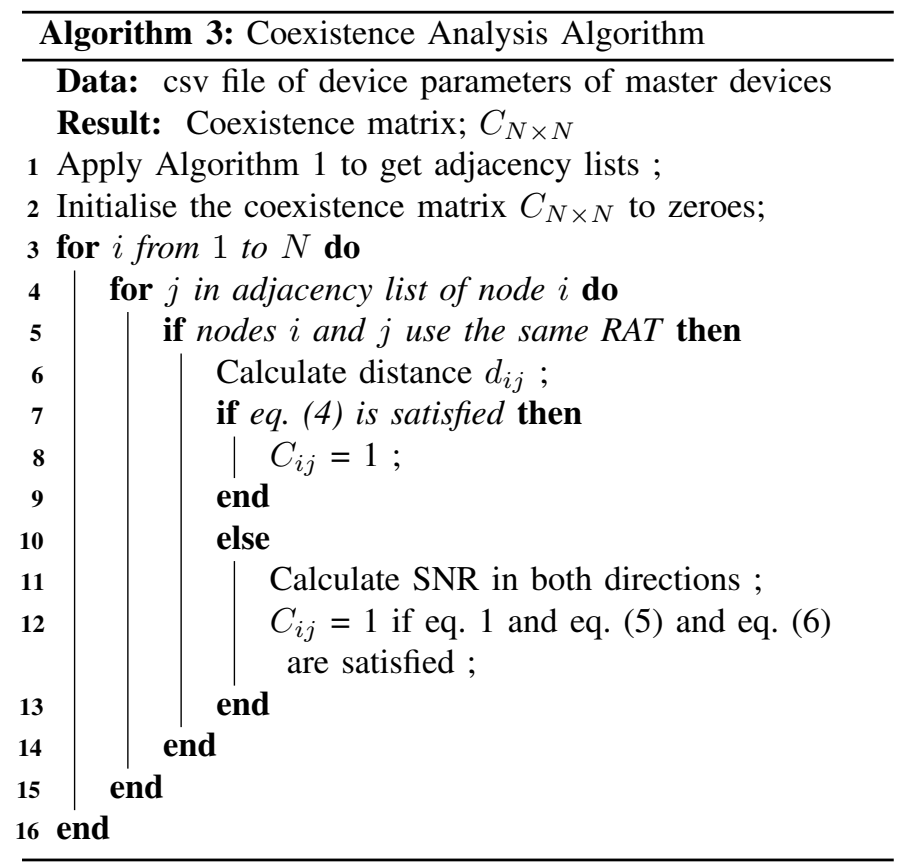

Then finally, the information from the coexistence matrix is then processed to generate "coexistent subsets" such that:

- each node in the subset can coexist with every other node in the subset,

- the total COR of the elements of the subset is $\leq$ unity.

Coexistent subsets form hyperedges. To reduce complexity, intersection of hyperedges is avoided. The procedure for hyperedge construction is summarised in Algorithm 4.

\section{B. Hyperedge Contraction}

Hyperedge contraction theory is then applied to decompose the hypergraph $H$ and make it amenable to the vertex colouring algorithm, without losing coexistence information. The output is a minor graph $H^{\prime}$ in which the hyperedges are contracted to single vertices and therefore resembles a traditional graph. The procedure is summarised in Algorithm 5 .

\section{Channel Allocation Algorithm}

This involves applying the vertex colouring algorithm to the minor graph and then applying the resultant colour map to the hypergraph, while ensuring that the vertices of a hyperedge get the colour that is assigned to the vertex that replaced the hyperedge in the minor graph. However, it was noted during algorithm testing that applying the vertex colouring algorithm to the minor graph may in some cases not exhaust all possible channel allocations. In hyperedge contraction, all vertices in a hyperedge are replaced by a single vertex such that all edges that were incident on the individual vertices are now incident on the new vertex, hence information about some possible
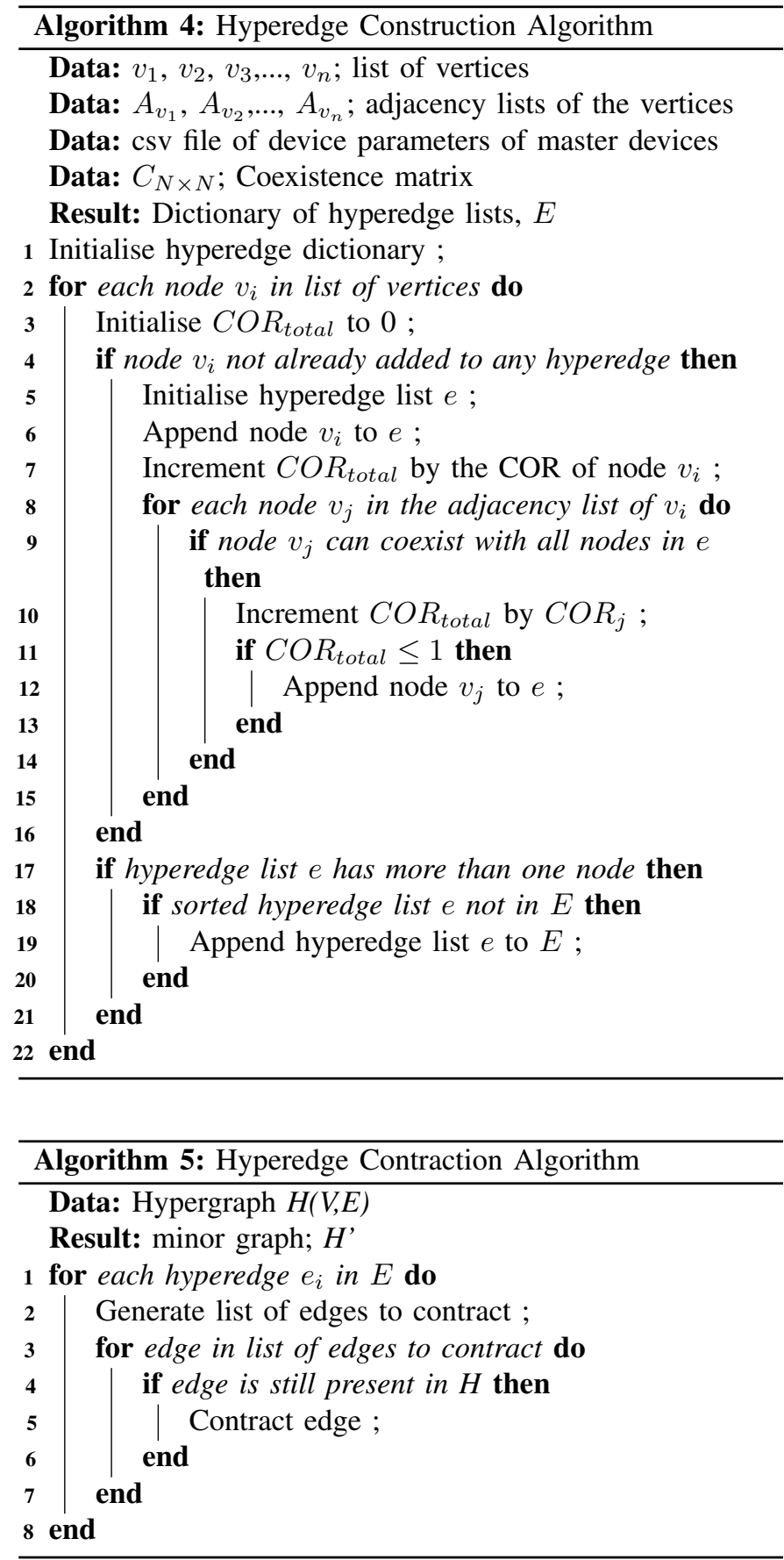

channel allocations could be masked. For instance, in Fig. 4, when hyperedge 11 is contracted, there will be an edge between the new vertex and $B S_{4}$. However $B S_{4}$ can share the same channel with $B S_{3}$ if they are able to coexist and calculation of COR will only involve $B S_{3}$ and $B S_{4}$ because $B S_{4}$ is not visible to $B S_{1}$ and $B S_{2}$. Hence, the algorithm was augmented to check for additional possible allocations that do not violate interference, coexistence and COR constraints if there are still some uncoloured nodes after vertex colouring of the minor graph. The entire procedure is summarised in Algorithm 6. 


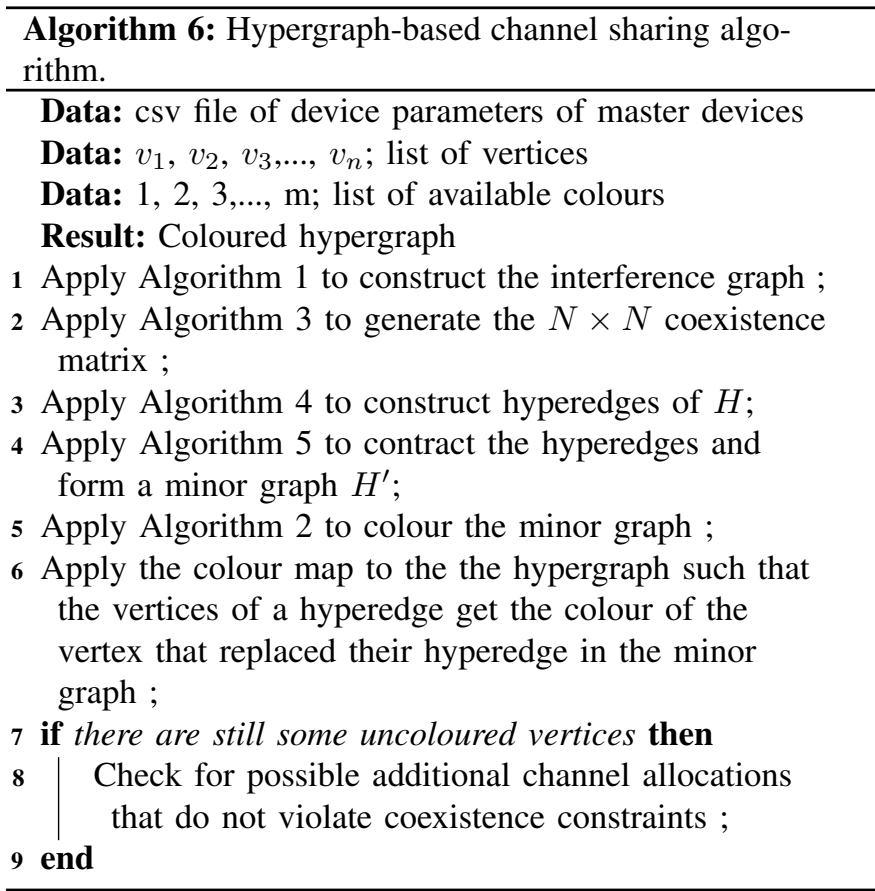

\section{Simulation Results}

The algorithms were implemented using the Python programming language. A $50 \mathrm{~km}$ by $50 \mathrm{~km}$ test area is considered. The test environment consists of three kinds of network cells: IEEE 802.11 outdoor WLAN with service radius of $1 \mathrm{~km}$, IEEE 802.22 WRAN with service radii of $5 \mathrm{~km}$ and $10 \mathrm{~km}$, which are randomly and independently distributed in the test area. The total number of networks, $N$ is 100 , in the ratio of 40:35:25 for $1 \mathrm{~km}-, 5 \mathrm{~km}$ - and $10 \mathrm{~km}$-coverage radius network. The rest of the test parameters are given in Table I.

The following propagation model, which is suggested in the IEEE 802.19.1 standard, is used to predict the path loss of the transmissions.

$$
L(d)=10 \log \left(\frac{4 \pi d}{\lambda}\right) \alpha-20 \log \left(h_{t} * h_{r}\right)
$$

TABLE I

PARAMETERS FOR SIMULATION

\begin{tabular}{|l|l||l||l|}
\hline Radio Access Technology (RAT) & $\begin{array}{l}802.11 \\
\text { TVHT }\end{array}$ & $\begin{array}{l}802.22 \\
\text { WRAN }\end{array}$ & $\begin{array}{l}802.22 \\
\text { WRAN }\end{array}$ \\
\hline \hline Cell coverage (m) & 1,000 & 5,000 & 10,000 \\
\hline BS / AP conducted power (dBm) & 15 & 20 & 31 \\
\hline BS/AP antenna gain (dB) & 5 & 5 & 5 \\
\hline CPE/UE antenna gain (dB) & 0 & 11 & 11 \\
\hline BS/AP antenna height (m) & 10 & 12 & 15 \\
\hline CPE/UE antenna height (m) & 1.5 & 6 & 10 \\
\hline Receiver sensitivity (dBm) & -88 & -88 & -88 \\
\hline Attenuation factor, $\alpha$ & 4 & 4 & 4 \\
\hline Channel Occupancy Rate (COR) & 0.3 & 0.25 & 0.25 \\
\hline SINR/SNR threshold (dB) & 10 & 10 & 10 \\
\hline Centre frequency (MHz) & 474 & 474 & 474 \\
\hline
\end{tabular}

A total of 100 simulations were performed to compare average performance of the hypergraph-based channel allocation algorithm with that of the graph-based exclusive channel allocation algorithm. The performance metric used is the average percentage of networks that are operational, having been assigned an operating channel.

\section{A. Average Percentage of Operational Networks as a Function of Number of Available Networks}

The objective of this test is to study the improvement in spectrum utilisation that is realised through channel sharing using the proposed algorithm when the number of available channels, $M$, is not sufficient for exclusive channel allocation. The results are given in Fig. 5.

It is assumed that there are 40 networks of $1 \mathrm{~km}$-radius in the test area at the start of the experiment. At $N=41$, the 5 $\mathrm{km}$-radius networks start to join. Performance of both models starts to drop from $100 \%$, but the rate of decline is higher for the graph-based exclusive channel allocation algorithm than the hypergraph-based channel sharing method. The vertices that represent the $5 \mathrm{~km}$-radius networks are likely to have a higher vertex degree than the $1 \mathrm{~km}$-radius networks since the 5 $\mathrm{km}$-radius networks are likely to have a higher average number of neighbours per network than the $1 \mathrm{~km}$-radius networks. Since the graph-based method assigns channels in the order of decreasing vertex degree, some $1 \mathrm{~km}$ radius networks start to lose their operating channels to the $5 \mathrm{~km}$-radius networks. Therefore the the rate of performance decline of the graph method increases as $N$ increases, whereas in the hypergraphbased model, the rate of performance decline decreases as $N$ increases because, as more $5 \mathrm{~km}$-radius networks join, they are likely to be assigned the same channel to share. At $N=75$, performance of the hypergraph-based model is higher than that of the graph-based model by $8 \%$ and $10 \%$ when $M=2$ and $M=3$, respectively.

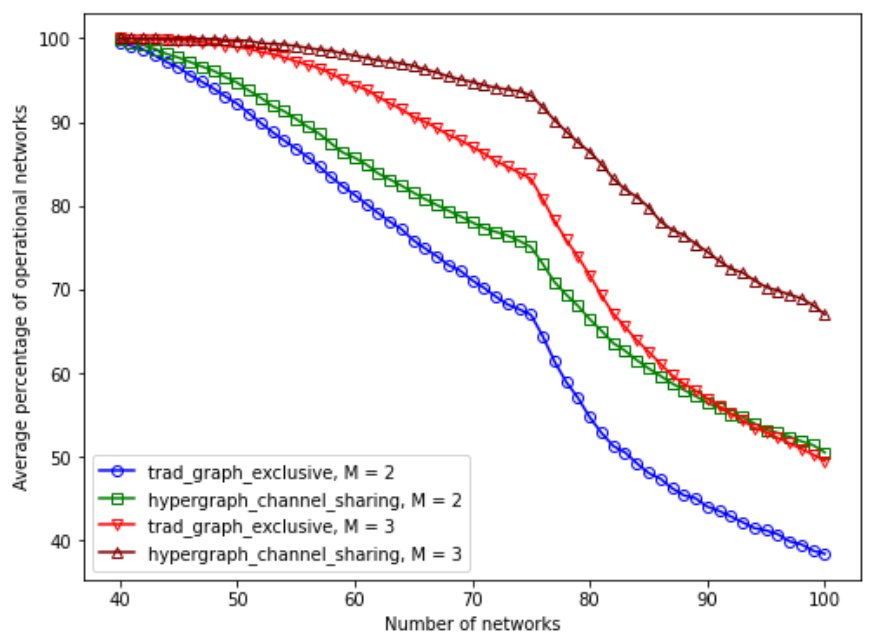

Fig. 5. Average percentage of operational networks as the number of networks is increased. 
Another sharp decline in performance for both models is noticed when the $10 \mathrm{~km}$-radius network start to join at $N=76$. The presence of the long-range networks increases the average number of neighbours per network and the number of available channels becomes significantly insufficient. But the hypergraph-based model still maintains better average performance, owing to efficient modelling of coexistence information for channel sharing. When $N=100$, the hypergraphbased model achieves average percentage of operational networks that is $12 \%$ and $17.5 \%$ higher than that of the traditional graph-based model when $M=2$ and $M=3$, respectively.

\section{B. Percentage of Operational Networks as a Function of Average Number of Available Channels}

The objective of this test is to study the efficiency in spectrum allocation that is realised through channel sharing using the proposed algorithm as the number of available channels is increased, starting at 4 channels, while the number of networks is held at 100. The results are given in Fig. 6 . The results show that, while the exclusive channel allocation requires 16 channels to achieve $100 \%$ average percentage of operational networks, the channel sharing algorithm requires only 9 channels.

\section{CONCLUSiOn AND FUtURE WORK}

In this paper, hypergraph theory is proposed for modelling radio resource allocation that involves channel sharing since conventional graph theory is not sufficient to model multiple relations. An increase in the number of neighbours per network results in a decrease in the opportunities for spatial re-use, but evaluation of spectral coexistence for channel sharing among neighbour networks helps to improve spectrum utilisation. The hypergraph-based model therefore results in more efficient spectrum utilisation than the traditional graph-based model because information about coexistent neighbour networks is

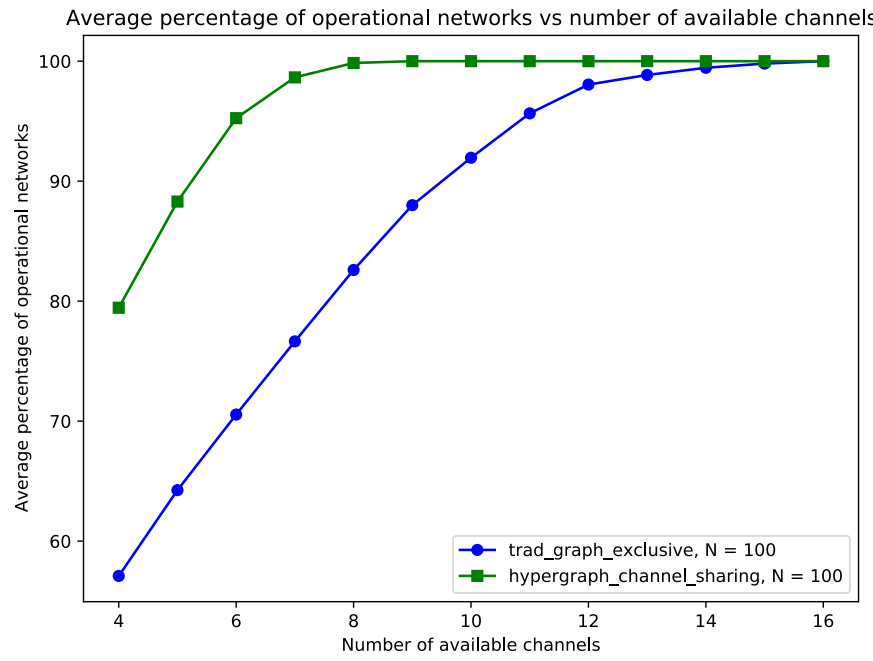

Fig. 6. Number of channels required to achieve $100 \%$ average operational networks. modelled for the channel allocation algorithm to utilise. Simulation results show that spectrum sharing using the hypergraphbased model increases the average spectrum utilisation by up to $17.5 \%$ and requires up to 6 less channels to achieve $100 \%$ average percentage of operational networks than spatial reuse alone. Having successfully implemented and tested the model, the road map for the further work is as follows. First, performance comparison of our model and the model that was proposed in [1] will be simulated. And finally, application of the model in coexistence management of GAA CBRS networks, including modelling of aggregate interference from coexistent groups, shall be investigated.

\section{ACKNOWLEDGMENT}

T.N. thanks the UK government, the Schlumberger Foundation and the University of Strathclyde for funding her studentship.

\section{REFERENCES}

[1] J. Wang, T. Baykas, S. Filin, M. A. Rahman, C. Song, and H. Harada, "Coexistence protocol design for autonomous decision-making systems in tv white space," in 2012 IEEE Wireless Communications and Networking Conference (WCNC), April 2012, pp. 3249-3254.

[2] S. Filin, F. Kojima, D. Noguet, J. Dore, B. Mawlawi, O. Holland, M. Z. Shakir, and H. Harada, "Ieee 1900.7 standard for white space dynamic spectrum access radio systems," in 2015 IEEE Conference on Standards for Communications and Networking (CSCN), Oct 2015, pp. 218-223.

[3] V. Voloshin, Introduction to Graph and Hypergraph Theory. New York: Nova Science Publishers, Incorporated, 2009.

[4] C. Chen, B. Wang, and R. Zhang, "Interference hypergraph-based resource allocation (ihg-ra) for noma-integrated v2x networks," IEEE Internet of Things Journal, vol. 6, no. 1, pp. 161-170, 2019.

[5] H. Zhang, L. Song, and Z. Han, "Radio resource allocation for deviceto-device underlay communication using hypergraph theory," IEEE Transactions on Wireless Communications, vol. 15, no. 7, pp. 48524861, 2016

[6] B. Wang, R. Zhang, C. Chen, X. Cheng, and L. Yang, "Interference hypergraph-based resource allocation (ihg-ra) for noma-integrated $\mathrm{v} 2 \mathrm{x}$ networks," in 2018 IEEE Global Communications Conference (GLOBECOM), 2018, pp. 1-6.

[7] J. Liu, S. Sun, J. Liu, and Y. He, "Hypergraph-based intercell interference coordination for qos guarantees in dense femtocell networks," in 2015 IEEE 81st Vehicular Technology Conference (VTC Spring), 2015, pp. 1-6.

[8] L. Kulacz, P. Kryszkiewicz, A. Kliks, H. Bogucka, J. Ojaniemi, J. Paavola, J. Kalliovaara, and H. Kokkinen, "Coordinated spectrum allocation and coexistence management in cbrs-sas wireless networks," IEEE Access, vol. 7, pp. 139294-139316, 2019.

[9] N. Wiebusch and U. Meier, "Evolutionary resource allocation optimization for wireless coexistence management," in 2018 IEEE 23rd International Conference on Emerging Technologies and Factory Automation (ETFA), vol. 1, 2018, pp. 1197-1200.

[10] G. Nychis, C. Tsourakakis, S. Seshan, and P. Steenkiste, "Centralized, measurement-based, spectrum management for environments with heterogeneous wireless networks," in 2014 IEEE International Symposium on Dynamic Spectrum Access Networks (DYSPAN), April 2014, pp. 303314.

[11] Ofcom. (2015, Feb.) Implementing tv white spaces. tvws-statement.pdf. [Online]. Available: https://www.ofcom.org.uk

[12] C. Sum, M. Zhou, L. Lu, F. Kojima, and H. Harada, "Performance and coexistence analysis of multiple ieee $802 \mathrm{wpan} /$ wlan/wran systems operating in tv white space," in 2014 IEEE International Symposium on Dynamic Spectrum Access Networks (DYSPAN), April 2014, pp. 145148. 